\title{
Diary ... Diary ... Diary ... Diary ... Diary ... Diary ... Diary ...
}

\subsection{December}

Multimedia Systems and Applications

Leeds, UK

Information: Mrs F J Johnson, Commercial Office, University of Leeds, Leeds LS2 9JT, UK.

Tel: 0532 336103, fax: 0532336107

\section{2-15 January}

BETT 94

Olympia, London, UK

Information: Emap, 12 Bedford Row, London WC1R 4DU, UK.

Tel: 0714044844

\section{2-6 February}

12th Expolangues

Paris, France

Information: OIP, 62, rue de Miromesnil, 75008

Paris, France.

\section{5-28 March}

\section{ALL Language World}

Southampton, UK

Information: Association for Language Learning.

Tel: 0788 546443, fax: 0788544149

\section{6-9 April}

IATEFL 28th Annual Conference

Brighton, UK

Information: IATEFL.

Tel: 0227276528 , fax: 0227274415

\section{1-13 April}

Teaching and Language Corpora 94

Lancaster, UK

Information: TALC94, Department of Linguistics, Lancaster University, Bailrigg, Lancaster LA1 4YT, UK.

Email: talc94@uk.ac.lancaster

\subsection{April}

XXIX Annual International Conference of the Association for Educational \& Training

Technology

Edinburgh, Scotland

Information: Guiti Saberi, Conference Administrator,
CASD, Napier University, 219 Colinton Road, Edinburgh EH14 1DJ, UK.

Tel: 031455 4306, fax: 031443 8161, email:

Denis@uk.ac.napier.dcs

14-17 April

TESOL '94, 'Sharing our Stories'

Baltimore, MA, USA

Information: TESOL US.

Tel: +1 703836 0774, fax: +1 7038367864

\section{9-23 April}

ALLC-ACH94 - Consensus ex machina

Paris, France

Information: A Salem/M Tournier, CNRS-INaLF, Lexicométrie et textes politiques, École Normale Supérieure, avenue de la Grille d'Honnuer, F-92211 Saint-Cloud, France.

Tel: +33 1 47719111, fax: +33146023911

\section{3-6 July}

SIGIR'94 - 17th International Conference on Research and Development in Information

Retrieval

Dublin, Ireland

Information: Dr A Smeaton, School of Computer Applications, Dublin City University, Glasnevin, Dublin 9, Ireland.

Tel: +3531 7045262, fax: +35317045442, email: asmeaton@ca.dcu.ie

\section{1-23 July}

Language Expo '94

Sydney, Australia

Information: Clare MacAdam, Fauth Royale \& Associates, 22/6 Avondale Road, Armadale, Victoria 3143, Australia.

Tel+61 3509 4160, fax: +61 35099115

\subsection{July}

World Conference on Computers in Education Birmingham, UK

Information: WCCE 95, Margaret Street, Birmingham B3 3BW, UK.

Tel: 021428 1258, fax: 0214282246 